\title{
Prosthesis-patient mismatch affects long-term survival after mechanical valve replacement
}

\author{
Shun Kohsaka, MD, ${ }^{\mathrm{a}, \mathrm{b}}$ Shaulnie Mohan, BA, ${ }^{\mathrm{a}, \mathrm{b}}$ Salim Virani, MD, ${ }^{\mathrm{a}}$ Vei-Vei Lee, MS, ${ }^{\mathrm{c}}$ Ariadna Contreras, MD, ${ }^{\mathrm{a}, \mathrm{b}}$ \\ George J. Reul, MD, d,e and Stephanie A. Coulter, MD ${ }^{\mathrm{a}, \mathrm{b}}$
}

Objective: We sought to examine the relationship between the degree of prosthesispatient mismatch and long-term survival after mechanical aortic valve replacement.

Methods: Prospectively collected long-term follow-up data from 469 consecutive patients who underwent aortic valve replacement between 1995 and 1998 were reviewed. The indexed effective orifice area was derived from the reference normal values of effective orifice area divided by the patient's body surface area. Outcome was stratified according to the severity of prosthesis-patient mismatch: moderate mismatch was defined as 0.65 to $0.85 \mathrm{~cm}^{2} / \mathrm{m}^{2}$ and severe mismatch as less than $0.65 \mathrm{~cm}^{2}$ / $\mathrm{m}^{2}$. The Cox proportional-hazards model with propensity score adjustment was used to adjust for the observed differences in baseline characteristics between the mismatch groups.

Results: The degree of prosthesis-patient mismatch was minimal in $57 \%$ of patients, moderate in $39 \%$, and severe in $4 \%$. Predictors of clinically significant mismatch included small aortic valve sizes (19 and $21 \mathrm{~mm}$ ), obesity, age greater than 65 years, and class III or IV heart failure. During a median follow-up period of approximately 7.9 years, overall survival was $77 \%$ in patients with minimal mismatch, $63 \%$ in those with moderate mismatch, and only $47 \%$ in those with severe mismatch $(P<.001)$. Moderate or severe mismatch was a significant predictor of poorer survival (hazard ratio, 1.6; 95\% confidence interval, 1.4-2.3; $P<.01$ ), even after adjustment for all significant clinical predictors (ie, propensity score; hazard ratio, $1.2 ; 95 \%$ confidence interval, $1.0-1.5 ; P=.05)$.

Conclusions: In a large aortic valve surgery population, prosthesis-patient mismatch occurred in $43 \%$ of patients, and those with significant mismatch had worse long-term outcomes than those with minimal mismatch.

From the Divisions of Cardiology, ${ }^{\text {a }}$ Biostatistics, ${ }^{c}$ and Cardiovascular Surgery, ${ }^{\mathrm{d}}$ the Texas Heart Institute at St Luke's Episcopal Hospital, Houston, Tex; and the Divisions of Cardiology ${ }^{\mathrm{b}}$ and Cardiovascular Surgery, ${ }^{\mathrm{e}}$ Baylor College of Medicine, Houston, Tex.

Received for publication Sept 4, 2007; revisions received Nov 2, 2007; accepted for publication Nov 13, 2007.

Address for reprints: Shun Kohsaka, MD, MC 1-133, PO Box 20345, Houston, TX 77225-0345 (E-mail: sk2798@columbia. edu).

J Thorac Cardiovasc Surg 2008;135:107680

\section{$0022-5223 / \$ 34.00$}

Copyright $\odot 2008$ by The American Association for Thoracic Surgery

doi:10.1016/j.jtcvs.2007.11.032
$\mathrm{P}$ rosthesis-patient mismatch (PPM) occurs when a prosthetic valve implanted during aortic valve replacement (AVR) is insufficient for a patient despite normal prosthesis function. ${ }^{1}$ The degree of PPM is defined by the effective orifice area (EOA) of the valve indexed to body surface area (BSA). Hemodynamic compromise occurs when indexed EOA is less than $75 \%$ of native EOA, leading to high postoperative transvalvular gradients and reduced regression of left ventricular hypertrophy. ${ }^{2}$ Significant mismatch has been reported in up to $60 \%$ of patients undergoing mechanical AVR.

However, the effect of PPM on survival remains controversial, possibly because the methods used to measure native EOA vary among studies. In some studies PPM has been shown to be an independent predictor of short-term survival in patients who have undergone AVR. ${ }^{3}$ Limited data suggest that the persistence of high gradients and limited regression might be an obstacle to improvement and might adversely affect both long-term survival and functional quality of life. ${ }^{2,4}$ We sought to determine whether PPM is associated with significantly reduced long-term survival in patients undergoing AVR. 

Abbreviations and Acronyms
$\mathrm{AVR}=$ aortic valve replacement
$\mathrm{BSA}=$ body surface area
$\mathrm{CI}=$ confidence interval
$\mathrm{EOA}=$ effective orifice area
$\mathrm{HR}=$ hazard ratio
$\mathrm{PPM}=$ prosthesis-patient mismatch
$\mathrm{OR}=$ odds ratio

\section{Materials and Methods}

Clinical, operative, and outcome data were prospectively collected from the Texas Heart Institute Research Database (THIRDBase), a comprehensive, longitudinal clinical registry of outcomes for more than 150,000 patients treated for cardiovascular disease at the Texas Heart Institute at St Luke's Episcopal Hospital. THIRDBase includes a wide range of data on all patients admitted to our institution with a diagnosis of cardiovascular disease. Written informed consent was obtained at hospital admission from all registered patients, and the study was approved by the institutional review board for the Texas Heart Institute at St Luke's Episcopal Hospital.

We examined records of 492 adult patients ( $\geq 18$ years old) who underwent mechanical AVR for aortic stenosis at the Texas Heart Institute from January 1, 1993, through December 31, 1998, and agreed to participate in a telephone survey. In addition, non-Texas residents were excluded from the present study to obtain accurate long-term survival information from the state database. The decision to perform AVR was made by the treating physicians in conjunction with their patients. We excluded patients who had native or prosthetic endocarditis (defined as blood culture-positive or systemic sepsis with the clinical syndrome of infective endocarditis, culture-positive valvular vegetations, or diagnosed or suspected endocarditis that required antibiotic treatment; $n=21$ ) or concomitant procedures other than coronary artery bypass grafting $(n=2)$. The remaining 469 patients were included in the present study.

Patient histories were obtained by means of interview at hospital or clinic presentation and were entered prospectively into the database. Hypertension was defined as either blood pressure greater than $130 / 90 \mathrm{~mm} \mathrm{Hg}$ or the combination of a history of hypertension and current use of antihypertensive medications. Diabetes mellitus was defined as a fasting blood sugar level of greater than $125 \mathrm{mg} / \mathrm{dL}$ or the use of antidiabetic agents. Renal insufficiency was defined as a serum creatinine level of $2 \mathrm{mg} / \mathrm{dL}$ or greater. Obesity was defined as a body mass index of 30 or greater. Coronary artery disease was defined either as significant stenosis $(>50 \%)$ in the left main artery or in all 3 major epicardial vessels. Peripheral vascular disease, cerebrovascular disease, and chronic lung disease were determined from each patient's medical history before the admission date. Previous myocardial infarction was deemed to be present if the patient had a history of myocardial infarction, coronary intervention, or coronary artery bypass surgery for myocardial infarction or if there were significant $Q$ waves on the patient's surface electrocardiogram.

Patient size was represented by BSA, which was calculated from height and weight. The indexed EOA was derived from the reference normal values of EOA divided by the patient's BSA. The reference values for EOA were based on previously published data. ${ }^{3}$ The indexed EOAs for St Jude hemodynamic plus valves were obtained through direct communication with the manufacturer, and these numbers were found to be in concordance with previously published data. ${ }^{5,6}$ Outcome was stratified according to the severity of PPM: minimal mismatch was defined as an indexed EOA of greater than $0.85 \mathrm{~cm}^{2} /$ $\mathrm{m}^{2}$, moderate mismatch as an indexed EOA of 0.65 to $0.85 \mathrm{~cm}^{2} / \mathrm{m}^{2}$, and severe mismatch as an indexed EOA of less than $0.65 \mathrm{~cm}^{2} / \mathrm{m}^{2}$.

The study end point was all-cause mortality. Survivorship was determined from the Texas State Department of Vital Statistics database, hospital records, and telephone follow-up. The Department of Vital Statistics keeps records of all births and deaths that have occurred in Texas from 1903 to the present. Consequently, follow-up information was available for all of the patients enrolled in the present study.

Most patient characteristics and event rates were recorded as binary categorical variables (ie, present or not present) and reported as percentages. The Pearson $\chi^{2}$ test was used to analyze discrete variables, and the Student $t$ test was used to analyze continuous variables. Logistic regression models using a forward stepwise variable selection process were developed to determine which clinical and angiographic variables were associated with PPM.

Cumulative survival was estimated with the Kaplan-Meier method and compared between groups by using a log-rank test. Cox proportional-hazards regression models were developed to determine whether severe PPM was associated with survival after adjustment for potential confounders. The assumption of linearity was evaluated for the continuous measures by using restricted cubic spines. Adjusted analyses were performed for the study cohort overall and stratified by age and sex. Further analysis was conducted to control for selection bias related to PPM. Unconditional logistic regression was used to estimate a propensity score for each patient. This score was then incorporated into a proportional-hazards model as a continuous variable with all predictor variables significant at a 2-tailed nominal $P$ value of less than .20 in the univariate analysis. Hazard ratios (HRs) and corresponding 95\% confidence intervals (CIs) were computed with associated $P$ values. All analyses were performed with SAS 6.09 software (SAS Institute, Cary, NC) for the VAX/VMS operating system.

\section{Results}

The median follow-up period was approximately 7.9 years (interquartile range, 5.0-10.0 years) for the 469 patients included in the study. Overall, $31.8 \%$ of the cases were concomitantly performed with coronary artery bypass grafting.

During this era, St Jude standard valves (St. Jude Medical, St Paul, Minn) were the preferred valve and accounted for $90.2 \%$ of our implantations (Table 1). Nineteen- and 21$\mathrm{mm}$ valves were placed in $27.5 \%$ of patients. The degree of PPM was minimal in $57 \%$, moderate in $39 \%$, and severe in only $4 \%$ of patients. Seventy-five percent of severe mismatch cases occurred after implantation of smaller (ie, 19 and 21 $\mathrm{mm})$ mechanical aortic valves. This severe mismatch occurred in $11 \%$ of all patients who received $19-$ or $21-\mathrm{mm}$ mechanical valves.

We examined the patients' baseline demographics, both as a whole and according to the degree of PPM (Table 2). Patient variables more commonly associated with greater 
TABLE 1. Type and size of implanted mechanical valves

\begin{tabular}{|c|c|c|c|c|c|c|c|}
\hline & \multicolumn{6}{|c|}{ Size (mm) } & \multirow[b]{2}{*}{ Total $(n=469)$} \\
\hline & $19(n=16)$ & $21(n=113)$ & $23(n=126)$ & $25(n=111)$ & $27(n=90)$ & $29(n=13)$ & \\
\hline \multicolumn{8}{|l|}{ Type } \\
\hline Carbomedics & $2(0.4 \%)$ & $6(1.3 \%)$ & $8(1.7 \%)$ & $5(1.1 \%)$ & $2(0.4 \%)$ & $0(0 \%)$ & $23(4.9 \%)$ \\
\hline St Jude HP & $4(0.9 \%)$ & $19(4.1 \%)$ & $0(0 \%)$ & $0(0 \%)$ & $0(0 \%)$ & $0(0 \%)$ & $23(4.9 \%)$ \\
\hline St Jude STD & $10(2.1 \%)$ & $88(18.8 \%)$ & $118(25.2 \%)$ & $88(22.6 \%)$ & $13(18.8 \%)$ & $88(2.8 \%)$ & $423(90.2 \%)$ \\
\hline
\end{tabular}

$H P$, Hemodynamics plus; $S T D$, Standard.

degrees of mismatch included hypertension, increasing age, and higher BSA. Female sex was associated with more severe mismatch; however, this association is probably substantially confounded by the smaller prosthetic valve sizes often used in the female patients. No significant differences were found in other domains at baseline. Predictors of moderate or severe mismatch included small aortic valve sizes (19 and $21 \mathrm{~mm}$ ), with an odds ratio (OR) of 8.3 (95\% CI, 5.1-13.6; $P<.01)$; obesity, with an OR of 3.4 (95\% CI, 1.9-6.1; $P<.01)$; and age greater than 65 years, with an OR of 1.9 (95\% CI, $1.2-$ $3.0 ; P<.01)$.

Overall survival was $90 \%$ at 1 year, $79 \%$ at 5 years, and $64 \%$ at 12 years. Twelve-year survival was $77 \%$ in patients with minimal mismatch, $63 \%$ in those with moderate mismatch, and only $47 \%$ in those with severe mismatch (Figure 1). Patients with moderate or severe mismatch had significantly poorer survival than patients with minimal mismatch $(P=.0076)$.

In a univariate model moderate or severe mismatch was strong and significantly associated with long-term mortality (HR, 1.6; 95\% CI, 1.4-1.8; $P<.001)$ and remained an independent predictor after adjustment for patient age and sex (HR, 1.4; 95\% CI, 1.2-1.6; $P=.04$ ). In a propensity-adjusted model there was a borderline statistically significant relationship between moderate or severe mismatch and increased mortality (HR, 1.2; 95\% CI, 1.0-1.5; $P=.05$; Table 3).

TABLE 2. Demographics of patients and their prostheses according to degree of prosthesis-patient mismatch

\begin{tabular}{|c|c|c|c|c|c|}
\hline & \multirow[b]{2}{*}{ All patients $(n=469)$} & \multicolumn{3}{|c|}{ Degree of PPM } & \multirow[b]{2}{*}{$P$ value } \\
\hline & & Minimal (n = 267) & Moderate $(n=183)$ & Severe $(n=19)$ & \\
\hline \multicolumn{6}{|l|}{ Preoperative characteristics } \\
\hline Age (y) & $56.1 \pm 11.5$ & $53.6 \pm 12.5$ & $59.5 \pm 8.9$ & $59.1 \pm 9.3$ & $<.001$ \\
\hline Female sex $(\%)$ & 33.3 & 26.9 & 40.9 & 47.3 & .001 \\
\hline Ejection fraction (\%) & $49.2 \pm 13.5$ & $48.8 \pm 14.2$ & $49.6 \pm 12.3$ & $50.2 \pm 14.1$ & .84 \\
\hline Body surface area $\left(\mathrm{m}^{2}\right)$ & $2.0 \pm 0.2$ & $1.9 \pm 0.2$ & $2.0 \pm 0.2$ & $2.1 \pm 0.2$ & .006 \\
\hline \multicolumn{6}{|l|}{ Preoperative comorbidities } \\
\hline Hypertension (\%) & 47.3 & 42.6 & 54.6 & 57.9 & .005 \\
\hline Insulin-dependent diabetes (\%) & 5.1 & 4.5 & 6.0 & 5.2 & .53 \\
\hline Dyslipidemia (\%) & 23.8 & 19.6 & 28.9 & 31.6 & .021 \\
\hline Smoking $(\%)$ & 44.3 & 44.5 & 44.2 & 42.1 & .86 \\
\hline Left main or 3-vessel disease $(\%)$ & 20.6 & 20.4 & 21.9 & 10.5 & .77 \\
\hline Unstable angina $(\%)$ & 18.1 & 16.4 & 20.2 & 21.1 & .30 \\
\hline Myocardial infarction (\%) & 2.4 & 3.0 & 1.6 & 0.0 & .24 \\
\hline NYHA heart failure class III or IV (\%) & 85.1 & 81.2 & 90.1 & 89.5 & .008 \\
\hline LVEF $\leq 35 \%(\%)$ & 18.7 & 21.2 & 15.8 & 13.3 & .38 \\
\hline Cerebrovascular accident (\%) & 6.4 & 5.6 & 7.7 & 5.3 & .54 \\
\hline Chronic renal failure (\%) & 6.6 & 4.1 & 10.4 & 5.3 & .038 \\
\hline Chronic lung disease (\%) & 26.1 & 23.9 & 28.4 & 31.6 & .23 \\
\hline Obesity (\%) & 14.9 & 8.6 & 21.9 & 36.8 & $<.001$ \\
\hline \multicolumn{6}{|l|}{ Operative characteristics } \\
\hline Urgent surgical intervention (\%) & 7.5 & 6.7 & 7.7 & 15.8 & .013 \\
\hline Concomitant CABG (\%) & 31.8 & 29.6 & 35.5 & 26.3 & .40 \\
\hline Clamp time (min) & $51.2 \pm 25.3$ & $48.0 \pm 21.5$ & $55.1 \pm 26.5$ & $59.4 \pm 50.8$ & .006 \\
\hline Small valve size: $19-21 \mathrm{~mm}(\%)$ & 27.5 & 10.5 & 47.5 & 73.7 & $<.001$ \\
\hline
\end{tabular}

PPM, Prosthesis-patient mismatch; NYHA, New York Heart Association; $L V E F$, left ventricular ejection fraction; CABG, coronary artery bypass grafting. * Minimal mismatch, Indexed effective orifice area greater than $0.85 \mathrm{~cm}^{2} / \mathrm{m}^{2}$; moderate mismatch, indexed effective orifice area of 0.65 to $0.85 \mathrm{~cm} / \mathrm{m}^{2} ;$ severe mismatch, indexed effective orifice area of less than $0.65 \mathrm{~cm}^{2} / \mathrm{m}^{2}$ ). 


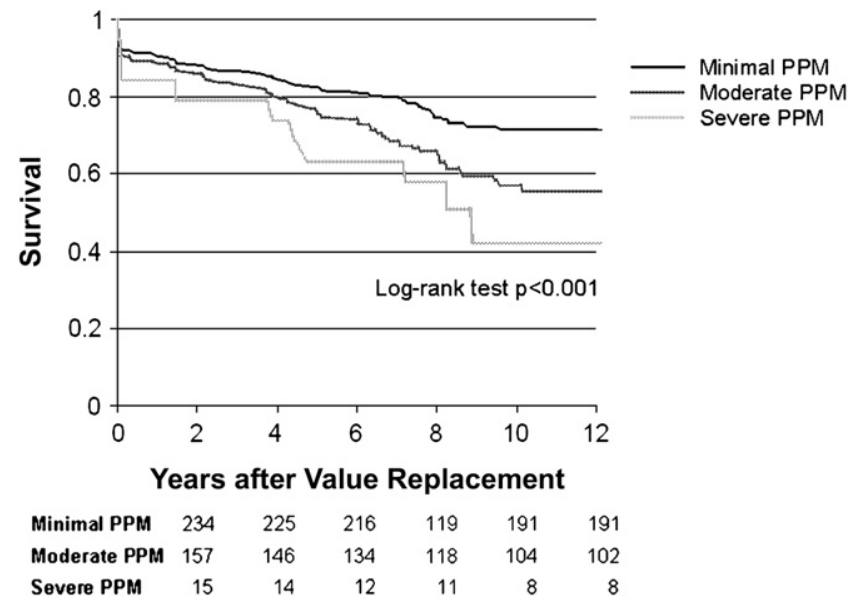

Figure 1. Long-term survival after aortic valve replacement by degree of prosthesis-patient mismatch (PPM). Minimal PPM, Indexed effective orifice area of greater than $0.85 \mathrm{~cm}^{2} / \mathrm{m}^{2}$ effective orifice area; moderate $P P M$, indexed effective orifice area of 0.65 to $0.85 \mathrm{~cm}^{2} / \mathrm{m}^{2}$; severe $P P M$, indexed effective orifice area of less than $0.65 \mathrm{~cm}^{2} / \mathrm{m}^{2}$.

Similarly, indexed EOA as a continuous variable was a significant predictor of long-term mortality in univariate analysis (HR, 0.3 ; 95\% CI, $0.2-0.5 ; P<.01$ ) but not in multivariate analysis (HR, $0.9 ; 95 \% \mathrm{CI}, 0.6-1.3 ; P=.54)$. Because the relationship between the indexed EOA and the risk of mortality was not linear, we also performed a logarithmic transformation of the indexed EOA and found a similar trend.

\section{Discussion}

Valve size in relation to BSA has been proposed as a predictor of survival after valve operations. In this study of 469 patients

TABLE 3. Risk-adjusted association of clinical and operative demographics with long-term mortality

\begin{tabular}{lcrr}
\hline \multicolumn{1}{c}{ Variable } & Hazard ratio & $\mathbf{9 5 \%} \mathbf{C l}$ & $\boldsymbol{P}$ value \\
\hline $\begin{array}{l}\text { Univariate model } \\
\text { Moderate or severe mismatch* }\end{array}$ & 1.6 & $1.4-2.3$ & $<.01$ \\
$\begin{array}{l}\text { Age- and sex-adjusted model } \\
\quad \text { Moderate or severe mismatch* }\end{array}$ & 1.4 & $1.2-1.6$ & .04 \\
$\begin{array}{l}\text { Propensity-adjusted model } \\
\text { Moderate or severe mismatch* }\end{array}$ & 1.2 & $1.0-1.5$ & .05 \\
$\quad$ LVEF $\leq 35 \%$ & 1.3 & $1.1-1.7$ & .01 \\
$\quad$ Urgent surgical intervention & 2.1 & $1.5-2.3$ & $<.01$ \\
Age >65 y & 1.9 & $1.5-2.3$ & $<.01$ \\
Chronic renal failure & 1.4 & $1.1-1.8$ & $<.01$ \\
$\quad$ Cerebrovascular accident & 1.6 & $1.2-2.2$ & $<.01$ \\
$\quad$ Left main or 3-vessel disease & 1.3 & $1.1-1.6$ & .01 \\
$\quad$ Diabetes & 1.6 & $1.3-2.0$ & $<.01$ \\
Smoking & 1.2 & $1.0-1.5$ & .01 \\
\hline
\end{tabular}

$\mathrm{Cl}$, Confidence interval; $L V E F$, left ventricular ejection fraction. ${ }^{*}$ Indexed effective orifice area of $0.85 \mathrm{~cm}^{2} / \mathrm{m}^{2}$ or less. with a median follow-up of 7.9 years, moderate or severe PPM (indexed EOA < 0.85) was associated with an increased risk of mortality in univariate analysis and with propensity score adjustment in multivariate analysis. The strengths of our study include a diverse patient population and long follow-up. Also, we included patients with several types of valve prostheses, and our population was representative of that seen in general clinical practice.

Previous short- and intermediate-term survival analyses have not consistently identified PPM as an independent predictor of adverse outcomes. ${ }^{7,8}$ It is therefore agreed that severe PPM increases early mortality, whereas its effect on late results is less clear. Clinically, severe PPM appears to be associated with a higher incidence of late symptoms of heart failure and less regression of left ventricular hypertrophy, as determined by means of echocardiographic analysis. ${ }^{9-11}$ Recently, an analysis from the Mayo Clinic identified severe PPM as an independent predictor of longterm mortality in patients with small aortic valve prostheses. ${ }^{12}$ However, it is important to note that only patients with small valves (19 and $21 \mathrm{~mm}$ ) were included in that study. Current mechanical valves provide low systolic gradients and acceptable orifice areas in patients with a small aortic root, but these devices necessitate anticoagulation, thus incurring a risk of hemorrhagic complication in older patients. ${ }^{13}$ The study also excluded all short-term deaths, which might have biased the results because short-term mortality is higher in patients with moderate or severe PPM. It is important to note that in our study patients undergoing aortic root enlargement concomitant with AVR were not excluded. In fact, throughout our study period, an attempt was made to upsize the aortic root to accommodate larger valve sizes. As a consequence, severe PPM was found in only $4 \%$ of patients.

Age greater than 65 years was an independent risk factor for long-term mortality in our patients. Because the population is aging, the incidence of degenerative aortic valve disease continues to grow, and the potential adverse effect of PPM on long-term survival in the elderly population is of increasing concern. Some studies have suggested that the effect of PPM is more important in younger patients ${ }^{14}$ and that elderly patients simply might not live long enough to manifest a survival decrement from significant PPM. ${ }^{15}$ However, we found no interaction between age and PPM. Instead, PPM predicted long-term mortality regardless of patient age.

Prosthesis size in our population was defined by the in vivo functional dimensions of the prosthesis and not by geometric dimensions. Geometric dimensions are usually obtained from the valve size reported on the label by the manufacturer, but this number variably refers to the diameter of the external sewing ring and to the diameter of the internal orifice. Pibarot and Dumesnil ${ }^{2}$ have suggested that rather than using geometric prosthesis dimensions, projected EOA, which is computed from in vivo EOA, should be used because it is a more physiologic measure. 
In the current study the association between moderateto-severe PPM and long-term survival was only modestly significant after adjustment for age and sex $(P=.05)$ and after stepwise modeling with propensity scoring $(P=.04)$. One possible explanation for this finding is that our study only included 469 patients, which might not have been enough to adequately examine 20 variables. Thus our results might underestimate the true independent association between substantial PPM and long-term outcomes. It is quite possible that if we had more patients, the association between moderate-to-severe PPM and long-term survival would become more significant.

Our study has other limitations. First, this was not a randomized trial; therefore surgical procedures were performed at the discretion of the individual surgeon, and patient characteristics in the 3 mismatch groups were intrinsically different. Propensity score adjustment was used to reduce treatment selection bias, allowing for a fairer comparison. Yet without treatment randomization, the possibility that there were unmeasured confounding variables cannot be excluded. The small number of patients in the severe mismatch group also limits our ability to draw firm conclusions about such patients or other types of patients with smaller valves.

Second, more than $90 \%$ of the valves used in our cohort were older St Jude Medical models, and the newer prostheses have better hemodynamic performance and are associated with a lower prevalence and severity of PPM, which might translate into better outcomes. Thus our results might not be generalizable to patients with newer prosthetic valves.

Third, although they have been validated multiple times in the past, ${ }^{2,16}$ most of our "reference values" for aortic valve prosthesis EOA were derived from the results of a single study and were therefore crucially dependent on the methods used in that study.

Finally, our models do not include the cause of death, and thus our models might be based on mortality unrelated to aortic valve disease. However, all-cause mortality is an appropriate end point to follow because it accounts for both cardiac and systemic disease and is unaffected by the reporting and misclassification bias potentially introduced by a physician's filing of a death report. ${ }^{17}$

In conclusion, in a large aortic valve surgery population, substantial PPM occurred in $43 \%$ of patients. Moderate or severe mismatch was most likely to occur in patients with larger BSA, older age, and smaller prosthesis size. The patients with substantial mismatch had significantly worse long-term outcome than the patients with minimal mismatch.

Stephen N. Palmer, PhD, ELS, contributed to the editing of this article. We thank Philippe Pibarot, DVM, PhD, for his expert opinion on our manuscript.

\section{References}

1. Rahimtoola SH. The problem of valve prosthesis-patient mismatch. Circulation. 1978;58:20-4.

2. Pibarot P, Dumesnil JG. Hemodynamic and clinical impact of prosthesis-patient mismatch in the aortic valve position and its prevention. J Am Coll Cardiol. 2000;36:1131-41.

3. Blais C, Dumesnil JG, Baillot R, Simard S, Doyle D, Pibarot P. Impact of valve prosthesis-patient mismatch on short-term mortality after aortic valve replacement. Circulation. 2003;108:983-8.

4. Tasca G, Brunelli F, Cirillo M, DallaTomba M, Mhagna Z, Troise G, et al. Impact of valve prosthesis-patient mismatch on left ventricular mass regression following aortic valve replacement. Ann Thorac Surg. 2005;79:505-10.

5. Vitale N, Caldarera I, Muneretto C, Sinatra R, Scafuri A, Di Rosa E, et al. Clinical evaluation of St Jude Medical Hemodynamic Plus versus standard aortic valve prostheses: the Italian multicenter, prospective, randomized study. J Thorac Cardiovasc Surg. 2001; 122:691-8.

6. Ismeno G, Renzulli A, De Feo M, Della Corte A, Covino FE, Cotrufo M. Standard versus hemodynamic plus 19-mm St Jude Medical aortic valves. J Thorac Cardiovasc Surg. 2001;121:723-8.

7. Hanayama N, Christakis GT, Mallidi HR, Joyner CD, Fremes SE, Morgan CD, et al. Patient prosthesis mismatch is rare after aortic valve replacement: valve size may be irrelevant. Ann Thorac Surg. 2002;73: $1822-9$.

8. Medalion B, Blackstone EH, Lytle BW, White J, Arnold JH, Cosgrove DM. Aortic valve replacement: is valve size important? J Thorac Cardiovasc Surg. 2000;119:963-74.

9. Ruel M, Rubens FD, Masters RG, Pipe AL, Bedard P, Hendry PJ, et al. Late incidence and predictors of persistent or recurrent heart failure in patients with aortic prosthetic valves. J Thorac Cardiovasc Surg. 2004;127:149-59.

10. Ruel M, Al-Faleh H, Kulik A, Chan KL, Mesana TG, Burwash IG. Prosthesis-patient mismatch after aortic valve replacement predominantly affects patients with preexisting left ventricular dysfunction: effect on survival, freedom from heart failure, and left ventricular mass regression. J Thorac Cardiovasc Surg. 2006;131:1036-44.

11. Tasca G, Mhagna Z, Perotti S, Centurini PB, Sabatini T, Amaducci A, et al. Impact of prosthesis-patient mismatch on cardiac events and midterm mortality after aortic valve replacement in patients with pure aortic stenosis. Circulation. 2006;113:570-6.

12. Mohty-Echahidi D, Malouf JF, Girard SE, Schaff HV, Grill DE, Enriquez-Sarano ME, et al. Impact of prosthesis-patient mismatch on long-term survival in patients with small St Jude Medical mechanical prostheses in the aortic position. Circulation. 2006;113: 420-6.

13. Jamieson WR, Miyagishima RT, Grunkemeier GL, Germann E, Henderson C, Lichtenstein SV, et al. Bileaflet mechanical prostheses for aortic valve replacement in patients younger than 65 years and 65 years of age or older: major thromboembolic and hemorrhagic complications. Can J Surg. 1999;42:27-36.

14. Moon MR, Pasque MK, Munfakh NA, Melby SJ, Lawton JS, Moazami N, et al. Prosthesis-patient mismatch after aortic valve replacement: impact of age and body size on late survival. Ann Thorac Surg. 2006;81:481-8.

15. Blackstone EH, Cosgrove DM, Jamieson WR, Birkmeyer NJ, Lemmer JH Jr, Miller DC, et al. Prosthesis size and long-term survival after aortic valve replacement. J Thorac Cardiovasc Surg. 2003;126: 783-96.

16. Pibarot P, Dumesnil JG, Cartier PC, Metras J, Lemieux MD. Patientprosthesis mismatch can be predicted at the time of operation. Ann Thorac Surg. 2001;71(suppl):S265-8.

17. Lauer MS, Blackstone EH, Young JB, Topol EJ. Cause of death in clinical research: time for a reassessment? J Am Coll Cardiol. 1999; 34:618-20. 